\section{Essere, storia e misericordia: l'oikonomia nella discussione tra Gregorio di Nissa e Eunomio*}

\section{Giulio Maspero}

Pontificia Università della Santa

Croce di Roma, Italia

http://orcid.org/0000-0001-6827-4436

\section{Orlando Solano Pinzón}

Pontificia Universidad Javeriana

http:/ / orcid.org/0000-0003-4446-626X

RICEVUTO: 14-11-17. APPROVATO: 14-03-18

Sommario: Lo studio percorre gli usi del termine oikonomia e dei suoi derivati nelle opere di Gregorio di Nissa dedicate alla discussione con Eunomio. La prospettiva analitica permette di ricostruire teologicamente la valenza ontologica della misericordia dalla prospettiva del rapporto tra essere e storia. L'attualità costante del pensiero cristiano può, infatti, essere rinvenuta nella dimensione trinitaria del Dio cristiano, che in quanto caratterizzato da una relazionalità immanente si rivela come relazione nel Filiazione del Verbo che si fa carne, in modo tale da riversare nella storia stessa la propria vita. L'uso di oikonomia è, dunque, legato all'ontologia trinitaria del Nisseno.

Parole Chiave: Patrologia; Gregorio di Nissa; ontologia trinitaria; oikonomia; cristologia; Eunomio.
Being, History, and Mercy: The Oikonomia in the Discussion between Gregory of Nyssa and Eunomius

Aвstract: The study covers the uses of the term oikonomia and its derivatives in the works of Gregory of Nyssa dedicated to the discussion against Eunomius. The analytical perspective allows us theologically to reconstruct the ontological value of mercy from the perspective of the relationship between being and history. The constant actuality of Christian thought can, in fact, be found in the Trinitarian dimension of the Christian God, which as characterized by an immanent relationality is revealed as a relationship in the filiation of the Word who became flesh, in such a way as to pour into history itself the own life. The use of oikonomia is, therefore, linked to the Trinitarian ontology of the Nyssen.

Key Words: Patrology; Gregory of Nyssa; Trinitarian ontology; Oikonomia; Christology; Eunomio.

\section{Come CitARE:}

Maspero, Giulio y Orlando Solano. "Essere, storia e misericordia: l'oikonomia nella discussione tra Gregorio di Nissa e Eunomio". Theologica Xaveriana 186 (2018): 1-24. https://doi.org/10.11144/javeriana. tx68-186.esmld

${ }^{*}$ Articolo di riflessione.

${ }^{a}$ Autore di corrispondenza. E-mail: maspero@pusc.it 


\section{Introduzione}

La teologia del secolo XX è attraversata e trascinata dalla riflessione sul rapporto tra essere e storia: idealismo, esistenzialismo e approccio fenomenologico si sono confrontati sul terreno teologico per cercare di rendere ragione della capacità della teologia stessa di illuminare le questioni attuali. La domanda che qui si pone è come tale sfida sia stata vissuta dai Padri della Chiesa, i quali hanno liberamente messo mano alle diverse filosofie che caratterizzavano l'epoca da loro vissuta. In questo senso, l'articolo presenta gli usi del termine oikovo $\mu^{\prime} \alpha^{1}$ e dei suoi derivati in particolare nell'opera di Gregorio di Nissa dedicata alla discussione con Eunomio².

Senza pretesa alcuna di esaustività, l'esposizione ha come fine evidenziare l'architettura teologica che ha caratterizzato la sintesi tra essere e storia sviluppata da Gregorio di Nissa. Così, il punto di partenza sarà la filiazione come espressione della misericordia divina, cui farà seguito un riferimento alla relazione tra teologia ed economia. Quindi, si porrà l'accento sul tema dell'unione ipostatica e infine sarà presa in considerazione l'unità d'azione delle tre persone divine in la Trinità nella storia.

\section{Filiazione e misericordia}

Significativamente la prima ricorrenza nel Contra Eunomium ${ }^{3}$ della terminologia in esame fa riferimento al ministero episcopale: Gregorio descrive il valore di Basilio che si oppose al potere imperiale, quando il prefetto Modesto ${ }^{4}$ si dedicava a rimuovere dalle loro sedi i vescovi ortodossi, per istallarvi illegalmente vescovi eretici ad oltraggio

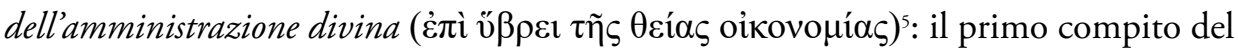
vescovo è la difesa della fede ortodossa, perché il suo insegnamento e il suo operato sono al servizio dell'economia divina. Così gli stessi trattati scritti contro Eunomio sono in primo luogo espressione della fedeltà di Gregorio al suo ministero, in aperto contrasto rispetto alla rottura di Ario con il vescovo Alessandro di Alessandria.

Nella discussione con il neoariano, il Nisseno ricorre anche al significato generico della terminologia in esame. Confutando l'affermazione di Eunomio che le

\footnotetext{
${ }^{1} \mathrm{Ci}$ si basa qui sulla ricostruzione presentata con maggiore dettaglio in Maspero, "Storia e salvezza: il concetto di oikonomia fino agli esordi del III secolo", 239-260.

${ }^{2}$ Su questo punto, si vedano: Dams, La controverse Eunoméenne; Cavalcanti, Studi Eunomiani; Kopecek, A History of Neo-arianism (due volumi); Vaggione, Eunomius. The Extant Works; Idem, Eunomius of Cyzicus and the Nicene Revolution; Pottier, Dieu et le Christ selon Grégoire de Nysse.

${ }^{3}$ Una prima ricorrenza appare nell'indice dell'opera: Contra Eunomium, Cap. 12, 3 (GNO I; 20,23-21,2).

${ }^{4}$ Vedasi N. ${ }^{\circ} 120$ a 152 di Moreschini, Gregorio di Nissa. Teologia trinitria. L'imperatore era in quel momento Valente, che morì nel 378.

${ }^{5}$ Per maggiori informazioni vedere: Gregorio di Nissa, Contra Eunomium I, 127, 12 (GNO I, 65, 21).
} 
medesime operazioni producono opere identiche, Gregorio afferma che, ad esempio, l'unica operazione del fuoco è quella di riscaldare, mentre il risultato è molteplice: il bronzo si fonde, la creta si indurisce, la cera si scioglie, e così via. Lo stesso dicasi del sole, che fa crescere alcune piante e dissecca le altre. In questa linea, passando

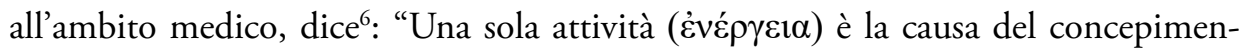

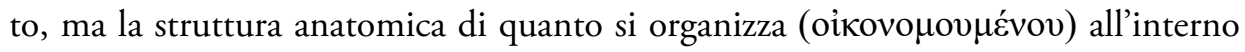
è multiforme, così che non si possono enumerare facilmente le qualità presenti nel corpo in base alla differenza"’

Dunque Gregorio sostiene, contro l'opinione dell'eretico, l'importanza del pensiero umano, in quanto è fonte delle conoscenze più elevate, come la geometria e l'aritmetica, la logica e la fisica, fino alla stessa filosofia, e praticamente "ogni occupazione dell'anima che riguardi le cose grandi e somme"s. In questo contesto ricorre al senso classico del termine oíkovouía, citando dopo l'agricoltura e la scienza della

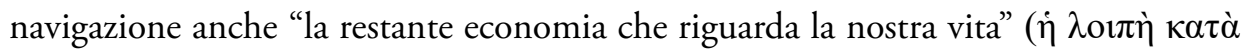

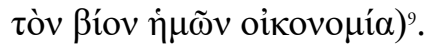

Per quanto riguarda gli usi propriamente teologici, invece, già l'esordio è significativo del tenore di tutta la discussione. Eunomio, infatti, sostiene che le sostanze sono necessariamente maggiori o minori nell'essere. La concezione filosofica ha sempre la priorità nel suo sistema di pensiero. Ben diverso è il modo di procedere nisseno:

Così che, per loro [cioè coloro che condividono l'opinione di Eunomio], sarebbe falsa anche la dottrina della Provvidenza e del Giudizio e dell'economia ( $\tau \tilde{\eta} \varsigma$ oíkovouías) e di tutto ciò che si è creduto che è stato fatto e per sempre viene fatto dall'Unigenito, poiché Egli verosimilmente si occuperebbe della cura del proprio bene, lasciando da parte il governo dell'universo. ${ }^{10}$

Gregorio parte invece dalla storia e da ciò che vede: considera l'agire pieno d'amore del Verbo nei confronti degli uomini. E se questi fosse inferiore al Padre, ciò significherebbe che é privo di qualche perfezione o bene. Questa deficienza lo spingerebbe a cercare di perfezionarsi così che non potrebbe occuparsi solo di noi, amarci disinteressatamente. L'oíkovouía è, dunque, essenzialmente misericordia e, in quanto tale, rivelazione dell'essere immanente di Dio. Invece l'unica ragione del fatto che

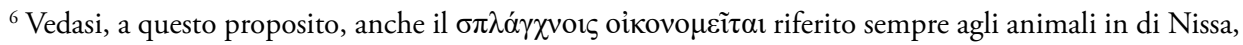
Contra Eunomium III, 8, 3, 7 (GNO II, 239, 3).

7 Ídem, Contra Eunomium I, 389, 1-4 (GNO I, 140, 26-141, 1).

${ }^{8}$ Ibíd., 181, 7-8 (GNO I, 277, 13-14).

${ }_{9}^{9}$ Di Nissa, Contra Eunomium II, 181, 9; idem, Contra Eunomium III, 2, 91, 8 (GNO II, 83, 5) il verbo è usato in senso generico per esprimere l'amministrare un colpo di fortuna.

${ }^{10}$ Ídem, Contra Eunomium I, 287, 8-13 (GNO I, 111,11-16).
} 
Dio ammetta la relazione con gli uomini è il suo amore per loro $(\varphi \imath \lambda \alpha v \theta \rho \omega \pi i ́ \alpha v)^{11}$ : siccome la sostanza che per sua natura è limitata non può raggiungere da sola la natura

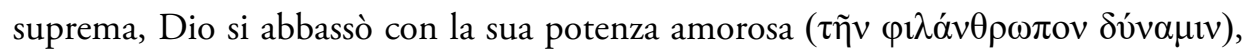
donando la sua grazia. Immagine cara a Gregorio per esprimere questa realtà è il sole con la luce dei suoi raggi:

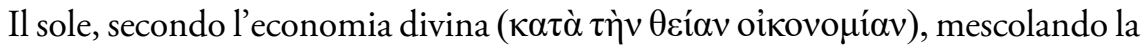
veemenza e l'ardore dei suoi raggi con l'aria intermedia, apporta una quantità di luce e di calore commisurata a coloro che li ricevono, mentre in sé il sole è inavvicinabile per la debolezza della nostra natura. ${ }^{12}$

Allo stesso modo la natura divina è inaccessibile per noi, ma si abbassa come una madre affettuosa sul suo piccolo. Non è dunque possibile proiettare gli effetti della condiscendenza amorosa di Dio sul suo essere:

E come non è possibile chiamare sordo colui che parla con un sordo a gesti, nel modo in cui questi per natura può capire, cos̀̀ nemmeno si può pensare che ci sia parola umana in Dio, per il fatto che ne fece uso per rivolgersi agli

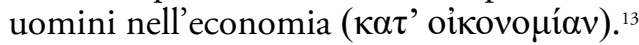

Lo stesso vale per noi quando gridiamo o fischiamo per richiamare gli animali: non è certo quello il nostro linguaggio. Così spesso la Sacra Scrittura attribuisce a Dio azioni e sentimenti umani, come l'adirarsi (Sal 105,40) e il pentirsi (1Sam 15,11), il dormire (Sal 77,65), lo star fermo, il muoversi e molte altre espressioni simili: "che per natura ( $\varphi v ́ \sigma \varepsilon \imath)$ non sono proprie di Dio, ma che non sono prive di utilità

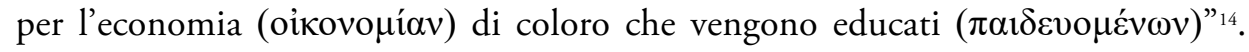
Il passaggio è estremamente breve, ma presenta l'interazione di tre concetti fondamentali: $\varphi v ́ \sigma ı \varsigma, ~ o i k o v o \mu i ́ \alpha$ e $\pi \alpha ı \delta \varepsilon i ́ \alpha$.

La discussione esegetica con Eunomio si sofferma, quindi, sull'interpretazione di Pro 8,22. Nella prima parte del versetto è detto che la Sapienza si edificò una dimora, cioè la carne del Signore, costruita proprio da un corpo virginale, perché la dimora non fosse estranea alla Sapienza stessa. La seconda parte si riferisce, invece, alla realtà resa una, costituita dalla casa e dalla Sapienza che edificò per sé la casa stessa, cioè dalla congiunzione dell'elemento umano e di quello divino, unitisi inscindibilmente nell'uom ${ }^{15}$. Quindi:

\footnotetext{
${ }^{11}$ Ídem, Contra Eunomium II, 417, 5-418, 1 (GNO I, 348, 10-12).

${ }^{12}$ Ibíd., 419, 1-5 (GNO I, 348, 17-21).

${ }^{13}$ Ibíd., 421, 1-4 (GNO I, 349, 17-20).

${ }^{14}$ Ídem, Contra Eunomium II, 424, 8-425, 1 (GNO I, 350, 20-21).

15 Ídem, Contra Eunomium III, 1, 45, 4-5 (GNO II, 19, 11-12).
} 
Si può osservare che è così anche nei Vangeli, dove il discorso, che è condotto secondo ciò che è conveniente al soggetto, significa l'elemento divino mediante l'indicazione più elevata e adatta a Dio, mentre significa l'elemento umano mediante quella inferiore e umile. Allo stesso modo anche in questa parte [del versetto] si può vedere che Salomone è mosso profeticamente e ha trasmesso

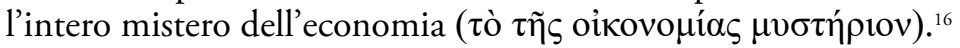

Per questo Salomone parla in primo luogo della potenza e delle operazioni della Sapienza che è anteriore ai secoli, riferendosi al suo ruolo nella creazione del mondo. Cioè inizia riferendosi ai suoi attributi divini. Poi: "Una volta spiegate queste e simili

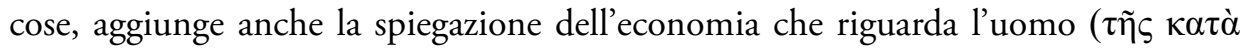

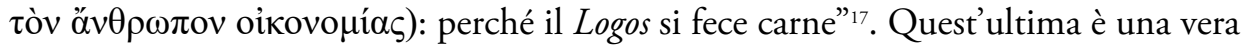

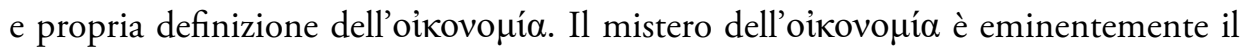
mistero dell'Incarnazione, in quanto mistero dell'Unigenito Figlio di Dio, Creatore dell'universo, che si fa creatura per rivelare ad ogni uomo l'amore del Padre.

Dio, infatti, non ha in sé nulla di creato e il termine creazione non si può applicare a nessuna delle realtà che sono in Dio: il Figlio non può essere creato, lui che è nel Padre, lui che è luce nella luce, vita nella vita e sapienza nella Sapienza. In parole del Nisseno:

Ma Colui che è nel seno del Padre (Gv 1, 18) non permette che mai si possa pensare il seno paterno vuoto di sé. Quindi Egli non è una delle cose generate dall'esterno nel seno [del Padre], ma, essendo la pienezza di ogni bene, viene pensato nel Padre, lui che è nel principio. ${ }^{18}$

La profondità del pensiero teologico di Gregorio presenta con estrema chiarezza anche in questo brano la distinzione tra creato ed increato: il mondo naturale è tutto ciò che si trova al di fuori del seno del Padre, tutto ciò che Dio crea e pone fuori di sé. Cristo, invece, pur essendo creato nella sua natura umana, è al tempo stesso vero Dio e non lascia mai il seno del Padre. Si vede come l'affermazione delle relazioni nella Trinità ha una base profondamente ontologica: Cristo è inseparabile dal Padre e dallo Spirito Santo anche nel suo peregrinare terreno poiché è della loro stessa natura, in quanto è Dio.

L'unione ipostatica è, dunque, il cammino voluto dal Padre e realizzato nel Figlio mediante lo Spirito Santo affinché ciò che fu posto fuori da Dio nell'atto creativo, possa ritornare all'intimità con il Padre. La profonda connessione cristologico-trinitaria,

\footnotetext{
${ }^{16}$ Ibíd.,1, 45, 7-46, 3 (GNO II, 19, 14-20).

${ }^{17}$ Ibíd., 1, 48, 1-3 (GNO II, 20, 8-10).

${ }^{18}$ Ibíd., 1, 49, 4-8 (GNO II, 20, 22-26).
} 
presentata in questo passo dal Nisseno, si dimostra fondamentale per la comprensione teologica dello schema exitus-reditus e, quindi, della teologia della storia del grande Cappadoce.

Gregorio continua nella sua esegesi cristologica del passo in questione:

Dunque l'espressione mi ha creato non è dell'elemento divino e puro, ma, come

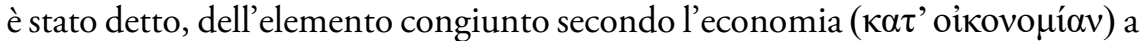
partire dalla nostra natura creata. Come è allora possibile che la stessa [Sapienza] possa fondare la terra e preparare i cieli e spalancare gli abissi, venendo chiamata Sapienza e Prudenza e Senso divino, e in questo passo venga creata a principio delle opere? E, come sta scritto, anche tale economia (oikovo $\mu$ í $\alpha$ ) viene realizzata non senza una grande causa. ${ }^{19}$

La sua ragione di essere consiste nel fatto che gli uomini rifiutarono con la disobbedienza la grazia ricevuta. Per questo Gregorio fa parlare la Sapienza in prima persona: spiega che ha accettato di venire creata, Lei che è da sempre e che non aveva alcun bisogno di essere creata, per riportare gli uomini allo stato primigenio. L'incarnazione è avvenuta radicalmente per amore degli uomini, come dice il Cristo, Sapienza di Dio: "Infatti, poiché fu corrotta la prima via, era necessario, per coloro che andavano errando, che fosse rinnovata ancora una volta una via nuova e vivente, cioè me stesso, che sono la Via (Gv 14,6)"20.

Per questo l'uomo deve rivestirsi di Cristo, Uomo nuovo, per tornare alla casa del Padre. Così Gregorio fonda la sua esegesi sull'affermazione paolina che Cristo è la Sapienza (1Cor 1,24); il lettore intelligente può allora giudicare da solo se è più appropriata alla Divinità la spiegazione di Eunomio o quella di Gregorio e chi è il più fedele alla norma della pietà:

Colui che dichiara che il Creatore ed il Signore dell'universo è stato creato e gli attribuisce onore uguale alla creazione che Gli serve, o piuttosto colui

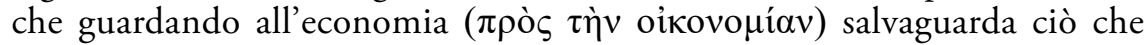
è conveniente alla nozione sia di divinità che di umanità. ${ }^{21}$

E la sequenza del ragionamento concorda con l'interpretazione spirituale ${ }^{22}$; infatti è giusto che prima venga il mi ha creato, poiché prima doveva nascere il bimbo per la nostra salvezza e solo poi essere chiamato con tutti i titoli messianici: "Dunque,

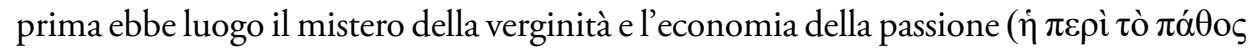
oíкоvo $\mu$ í $\alpha$ ) e allora i saggi architetti della fede gettarono il fondamento della fede" ${ }^{23}$.

\footnotetext{
${ }^{19}$ Ibíd., 1, 50, 1-51, 1 (GNO II, 21, 5-12).

${ }^{20}$ Ibíd., 1, 51, 11-13 (GNO II, 21, 22-24).

${ }^{21}$ Ibíd., 1, 54, 4-8 (GNO II, 22, 22-26).

${ }^{22}$ Ibíd., 1, 55, 1-2 (GNO II, 23, 2-3).

${ }^{23}$ Ibíd., 1, 55, 8-11 (GNO II, 23, 9-12).
} 
La fede si appoggia, dunque, sulla vita terrena di Cristo, dalla nascita fino alla morte: è su di essa che è stata fondata la formulazione della fede e costruito l'edificio della teologia. Lo stesso si ripete nella vita spirituale di ogni uomo: il fondamento è sempre la vita di Cristo. Per giungere alla divinizzazione bisogna, innanzitutto, che Egli nasca in noi: tutta la vita del cristiano è per Gregorio fondamentalmente cristica. È, infatti, la vita terrena del Figlio di Dio la norma ed il cammino perché ogni uomo diventi figlio di Dio.

L'economia e l'immanenza trinitarie sono unite proprio dall'unica Filiazione di Cristo dal Padre, che costituisce il suo stesso essere Persona, il suo modo di esistenza. La vita terrena di Cristo è, allora, spiegazione all'uomo di questa Filiazione. Spiegazione nel duplice senso di dispiegamento temporale e di educazione dell'uomo perché apprenda ad essere figlio. È nell'essere Figlio, nella Persona divina, dunque, che si uniscono le due nature. Per questo: "La parola Figlio rende testimonianza in lui ad entrambe le realtà: nell'uomo all'umanità e in Dio alla divinità" ${ }^{24}$.

Il ruolo peculiare del nome di Figlio è sottolineato ancor di più dal fatto che per Gregorio tutti gli attributi divini si suddividono in due gruppi ben distinti:

Ma è possibile dividere in due gruppi, secondo una certa arte e regola, il significato dei nomi divini. I primi, infatti, servono ad indicare la gloria somma e ineffabile, i secondi indicano la multiformità della sua provvidenziale economia

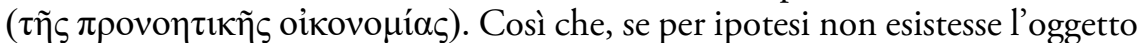
della sua beneficenza, nemmeno sarebbe stato possibile applicare a lui questi termini che indicano la beneficenza stessa. Ma quei nomi che esprimono ciò che è proprio di Dio, anche in assenza di coloro che sono oggetto dell'economia

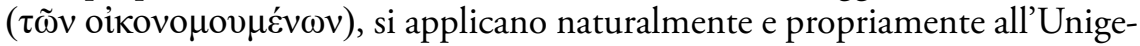
nito Dio. ${ }^{25}$

Così attributi come vite, pastore e medico appartengono al secondo gruppo di nomi, mentre Figlio appartiene al primo, insieme con potenza di Dio, destra di Dio, Logos ed Unigenito.

È estremamente importante sottolineare che questa suddivisione tra nomi economici e nomi teologici permette di parlare della seconda Persona stessa della Trinità, esprimendo la relazione tra il Padre e il Figlio. Per Gregorio, infatti, è inconcepibile affermare l'uguaglianza di natura senza considerare anche le relazioni intratrinitarie che costituiscono le tre Persone. Il lettore postcartesiano fatica a comprendere la profondità del pensiero nisseno, e patristico in generale, che non oppone mai natura e persona, ma anzi fonda reciprocamente la comprensione di una nell'altra.

${ }^{24}$ Ibíd., 1, 93, 7-9 (GNO, II, 35, 25-27).

${ }^{25}$ Ibíd., 1, 131, 7-132, 7 (GNO II, 48, 1-9). 
Così Gregorio scrive: "Ed è chiamato Figlio, Destra, Unigenito, Logos, Sapienza, Potenza e con tutti quegli appellativi che esprimono una relazione ( $\left.\pi \rho \operatorname{có}_{\varsigma} \tau \mathrm{\imath}\right)$, in quanto viene assolutamente denominato come in congiunzione relazionale con il Padre" ${ }^{26}$.

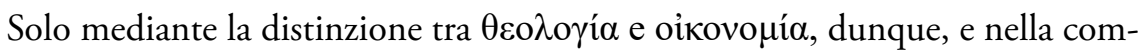
prensione profonda della filiazione divina, si può spiegare perché Cristo è detto sia Primogenito che Unigenito:

Ma come è detto Dio e uomo, Figlio di Dio e Figlio dell'Uomo, forma di Dio e forma di servo, essendo Dio, Figlio di Dio e forma di Dio secondo la natura superiore ed essendo divenuto uomo, Figlio dell'Uomo e forma di servo

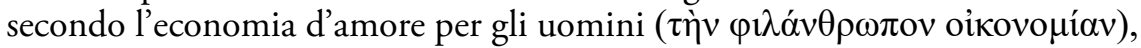
così anche lui che è l'Unigenito Dio diventa primogenito di tutta la creazione, lui che è Unigenito nel seno del Padre $(G v 1,18)$ e tra coloro che si salvano per la nuova creazione è divenuto e chiamato primogenito di tutta la creazione. ${ }^{27}$

È così che in Cristo si uniscono il tempo e l'eterno, il Creatore e la creatura, nel suo protendersi verso l'uomo nell'economia d'amore.

\section{Teologia ed economia}

In tale discussione assumono un'importanza fondamentale alcuni specifici passi della Sacra Scrittura, come At 2,36 e il prologo giovanneo, i quali esigevano una chiara e netta presa di posizione, che preservasse la distinzione tra creato e non creato. Si tratta quindi non di un elemento estrinseco, imposto dalle categorie di pensiero al dato scritturistico, ma di un'esigenza interna richiesta dalla comprensione del dato scritturistico stesso nel suo insieme.

Così sia Gregorio che Basilio dovettero affrontare la disputa con Eunomio sul discorso di Pietro in At 2,36:

Dunque Pietro disse ai Giudei che Dio ha costituito Signore e Cristo quel Gesù che voi avete crocifisso $($ At 2, 36). Ma noi diciamo che non è pio riferire il [verbo] ha costituito alla Divinità dell'Unigenito, mentre affermiamo che esso

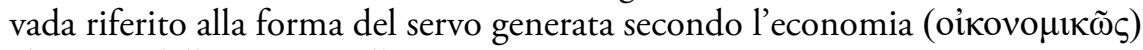
al tempo dell'avvento nella carne. ${ }^{28}$

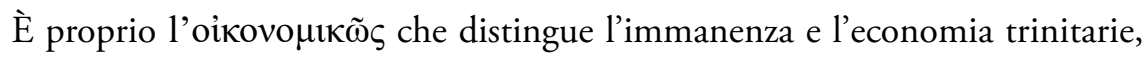
salvando la perfetta divinità consustanziale delle tre persone divine ${ }^{29}$. Eunomio riferisce,

\footnotetext{
${ }^{26}$ Ibíd., 133, 9-134, 1 (GNO II, 48, 19-22).

${ }^{27}$ Ibíd., 2, 55, 5-56, 1 (GNO II, 70, 22-71, 2).

${ }^{28}$ Ibíd., 3, 12, 3-8 (GNO II, 111, 14-19).

${ }^{29}$ Maspero, "Economy”, 541.
} 
invece, il verbo ha costituito alla dimensione che precede i secoli e cita Basilio (Adversus Eunomium 2, 3, PG 29, 576 D-577A) per confutarlo, il quale già si era opposto alla lettura eunomiana di At 2,36: il passo non si riferirebbe, secondo Basilio, alla sostanza del Verbo di Dio che era da principio presso Dio, ma a colui che si abbassò nella forma del servo e assunse nel corpo della nostra umiltà la nostra stessa natura e fu crocifisso a causa della debolezza. Quindi continua con la citazione, tratta dal II libro del Contra Eunomium di Basilio (PG 29, 576D-577A), parole di Basilio stesso ripetute nel corso della diatriba da Eunomio:

Chiunque presti anche solo un po' di attenzione al senso del testo apostolico può riconoscere che questo non ci tramanda una forma della teologia ( $\theta \varepsilon 0 \lambda$ oүías), ma propone le ragioni dell'economia (oikovouías). Infatti dice che Dio ha costituito Signore e Cristo quel Gesù che voi avete crocifisso (At 2,36), appoggiandosi chiaramente al pronome dimostrativo ( $\tau$ oṽ $\tau$ ov) per indicare il suo essere uomo e visibile a tutti. ${ }^{30}$

Eunomio contesta Basilio dicendo che, se Pietro non si riferisce al Logos che da principio era presso Dio, ma a colui che si rese visibile e annientò sé stesso (cioè all'umanità e non alla natura divina) e se annientò sé stesso nella forma di servo l'uomo visibile al fine di essere fatto uomo, si giungerebbe all'assurdità che un uomo si è annientato per divenire uomo. Continua Eunomio:

Ma contrasta con queste parole anche la stessa natura dei fatti e le contraddice chiaramente anche lo stesso che esalta con la sua teologia questa economia (oikovo $\mu i ́ a v$ ) [cioè Giovanni], poiché dice che non l'uomo visibile, ma lo stesso Verbo che era in principio e che era Dio assunse la carne, che equivale con altre parole a prendere la forma di servo. ${ }^{31}$

È chiara, in questi passi, la differente concezione del rapporto tra $\theta \varepsilon 0 \lambda$ oүía

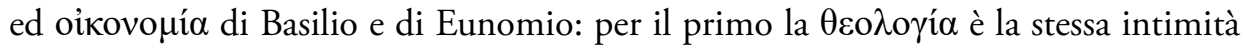
dell'eternità divina, per il secondo la $\theta \varepsilon o \lambda o \gamma i ́ \alpha$ è solo la comprensione e l'espressione umana della rivelazione, in quanto si muove in assenza di una netta separazione ontologica tra creato e Creatore.

In questo contesto l'equilibrio e la penetrazione teologica nissena del mistero dell'incarnazione si esprimono in tutta la loro forza:

Infatti noi diciamo che anche il corpo con il quale accettò la passione, unito alla

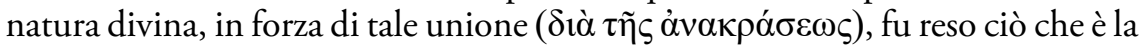
natura che lo assunse. E siamo tanto lontani dal pensare qualcosa di meschino a proposito del Dio Unigenito, che, anche se egli assunse qualcosa di inferiore

\footnotetext{
${ }^{30}$ Di Nissa, Contra Eunomium I, 3, 16, 10-7, 1 (GNO II, 113, 1-9).

${ }^{31}$ Ibíd., 3, 19, 1-7 (GNO II, 114, 11-17).
} 


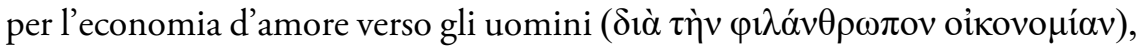
crediamo che perfino questo è stato trasformato in ciò che è divino e puro. ${ }^{32}$

È qui evidente che il doppio movimento che caratterizza la teologia di Gregorio, che si sviluppa in senso discendente -dalla Trinità alla creatura- per riportare quindi la creatura all'intimità delle tre persone divine, in un successivo movimento ascendente, si fonda sull'incarnazione stessa. Infatti, una volta messo in chiaro che è Dio stesso che si incarna, ora il Nisseno considera il momento dell'elevazione, nel quale la natura umana è resa divina in Cristo. Il realismo della concezione gregoriana è estremo. Cristologia e soteriologia sono inseparabili nell'unità di questo duplice movimento, che è come un sospiro d'amore per l'uomo della Trinità stessa. ${ }^{33}$

La penetrazione del mistero dell'incarnazione manifestata qui dal Nisseno rende possibile intravvedere il vero fondamento del valore divino dell'umano: tutto ciò che appartiene alla natura umana di Gesù -i suoi sentimenti, i suoi dolori, le sue azioni, in una parola, il suo cuore-sono resi divini ed eterni. È qui che la storia dell'uomo trova il cammino per l'eternità.

Sono la croce, la passione e il dolore che Eunomio non può accettare. La passione è indegna di Dio, ignominiosa. Essa è la prova dell'inferiorità della sostanza di Cristo rispetto a quella del Padre. Eunomio semplicemente rifiuta il mistero, mentre per Gregorio:

Nulla di ciò che si muove secondo la propria natura suscita meraviglia come per qualcosa di straordinario, ma è quanto supera i limiti della natura che diviene oggetto d'ammirazione più di ogni altra cosa e ad esso tende ogni orecchio e si protende ogni mente, colma di meraviglia per lo straordinario. ${ }^{34}$

La mente non può che meravigliarsi di fronte al mistero di Dio che entra nella storia facendosi uomo, per salvare l'uomo stesso e la sua storia.

Quindi, in Contra Eunomium III, 3, 49, 1 (GNO II, 125, 6), si riprende il principio esegetico fondamentale di Basilio, vera spina nel fianco del neoariano, che sarà citato ancora in Contra Eunomium III, 3, 59, 2 (GNO II, 128, 23): l'apostolo non parla di teologia, ma di economia. Eunomio insiste, tuttavia, nella sua replica, dicendo che l'interpretazione di Gregorio e Basilio porta ad affermare che un uomo

\footnotetext{
32 Ibíd., 3, 34, 1-7 (GNO II, 199, 21-27).

${ }^{33}$ Lo studio della cristologia nissena merita un serio approfondimento. Si vedano, ad esempio, i seguenti studi: Daley, "Heavenly Man and Eternal Christ: Apollinarius and Gregory of Nyssa on the Personnal Identity of the Savior" e idem, "Divine Transcendence and Human Transformation: Gregory of Nyssa's Anti-Apollinarian Christology".
}

${ }^{34}$ Di Nissa, Contra Eunomium, 3, 34, 12-35, 1 (GNO II, 120, 1-5). 
si è annientato per diventare uomo. E il Nisseno sembra quasi perdere la pazienza di fronte a tanta ostinazione:

Che hanno in comune una cosa con l'altra? Se diciamo che l'Apostolo non ci ha

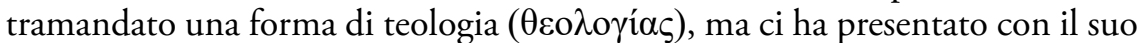

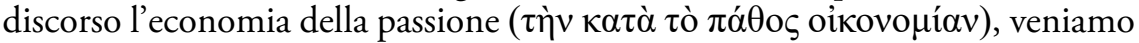
accusati per questo di parlare dell'annullamento di un uomo in un uomo, di una forma di servo preesistente dall'eternità e di dire che l'uomo nato da Maria sarebbe più antico della sua venuta nella carne? ${ }^{35}$

Gregorio vede così assurde queste accuse che dice superfluo il ribatterle e si limita a ritorcere contro Eunomio le sue stesse critiche: “...in quanto sono loro e non noi che insegnano il passaggio da simile a simile del Figlio nell'economia della passione

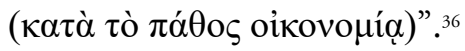

Infatti Eunomio fa del Verbo una sostanza creata: per questo è lui che afferma che il simile è stato annientato per essere simile, cioè una sostanza creata per passare ad essere un'altra sostanza creata. Sempre la distinzione fondamentale è quella tra creato ed increato.

E l'Unigenito ha creato ogni cosa, ed anche la natura umana è una delle sue opere. Questa si trova nella corruzione della morte per il fatto di essersi volta al male. Ma:

Nuovamente l'ha attratta per mezzo di se stesso alla vita immortale, assumendo in sé l'intera umanità mediante l'uomo nel quale prese dimora, e unì indissolubilmente la propria potenza vivificatrice alla natura mortale e peritura, e mediante l'unione con sé stesso trasformò il nostro stato mortale in grazia e potenza di vita. ${ }^{37}$

Il movimento ascendente assume la forma di una attrazione, che in Cristo si estende a tutta la natura umana, trasformando la morte dell'uomo in vita. È in questa attrazione che trova il suo compimento il mistero del tempo e della storia:

E diciamo che questo è il mistero della carne del Signore: Colui che è immutabile nasce nella realtà mutevole, affinché, mutandola in meglio e trasformandola dalla condizione peggiore, eliminasse dalla natura la malvagità mescolata alla condizione mutevole, distruggendo il male in sé stesso. ${ }^{38}$

Ecco cos'è, per Gregorio, il centro del mistero dell'incarnazione: l'eterno e immutabile è nato nel tempo per salvare l'uomo dal male, per trasformare il tempo

\footnotetext{
${ }^{35}$ Ibíd., 3, 49, 3-9 (GNO II, 125, 8-14).

${ }^{36}$ Ibíd., 3, 50, 10-51, 1 (GNO II, 125, 25-27).

${ }^{37}$ Gregorio di Nazianzo, Epistulae theologicae 101, 19, 1-2 (SCh 208, 44).

${ }^{38}$ Di Nissa, Contra Eunomium III, 3, 52, 1-6 (GNO II, 126, 9-14).
} 
e la storia dell'uomo da dentro, aprendo loro il cammino all'amore divino, nell'abbassamento per amore degli uomini dalla natura superiore alla natura più umile ${ }^{39}$.

\section{L'unione ipostatica}

Per questo tutto il discorso ruota attorno all'unione ipostatica. Gregorio si difende dall'accusa di parlare di due Cristi e di due Signori. Tutto il suo argomento si basa sul

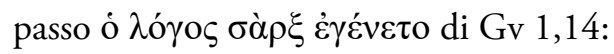

Infatti noi vediamo distintamente i fatti realizzati nell'economia della carne

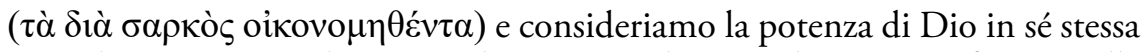
e così lui [Giovanni] dice in modo quasi simile a noi che si è manifestato nella carne il Logos che era in principio. ${ }^{40}$

Eppure nessuno accusa Giovanni di parlare di due Cristi. Infatti la sua affermazione fondamentale è proprio che il Logos manifestatosi nella carne è identico al Logos che era presso Dio ${ }^{41}$. Eppure, la carne non è da sempre unita alla Divinità: "Ma la carne non era identica alla Divinità prima di venire anch'essa trasformata nella Divinità, così che necessariamente alcune cose corrispondono al Logos Dio, altre alla forma del servo" ${ }^{42}$.

E proprio nella formulazione dell'inscindibile unione tra natura divina ed umana in Cristo, Gregorio raggiunge il vertici della sua cristologia, precorrendo di gran lunga i tempi ${ }^{43}$ :

La natura divina è sempre una e la medesima ed uguale a sé stessa, mentre la carne è di per sé ciò che la ragione ed i sensi ne conoscono. Ma una volta unita al Divino, [la carne] non rimane più nei suoi limiti e proprietà, ma viene assunta in ciò che è dominante e superiore, anche se la considerazione intellettuale ( $\dot{\eta} \theta \varepsilon \omega \rho$ pí $)$ delle proprietà della carne e della divinità rimane inconfusa ( $\dot{\alpha} \sigma 0 ́ \gamma \chi v \tau o \varsigma)$, fin tanto che ciascuna di esse viene considerata in sé. ${ }^{44}$

${ }^{39}$ Ibíd., 3, 52, 12-13 (GNO II, 126, 20-21). Per maggiori informazioni sul tema della relazione tra teologia ed economia vedasi: Brugarolas, "La mediación de Cristo en Gregorio de Nisa”, 306-313.

${ }^{40}$ Di Nissa, Contra Eunomium III, 3, 62, 1-4 (GNO II, 129, 23-26).

${ }^{41}$ Ibíd., 3, 62, 7-8 (GNO II, 130, 1-2).

${ }^{42}$ Ibíd., 3, 62, 8-63, 1 (GNO II, 130, 2-5).

${ }^{43}$ La cristologia nissena è stata spesso criticata dal punto di vista terminologico ed accusata sia di nestorianismo che di monofisismo (Tixeront, Histoire des dogmes dans l'antiquité chrétienne II, 128). J. R. Bouchet ha ampiamente dimostrato l'infondatezza di queste accuse: quando si considera il vocabolario cristologico nisseno nel contesto del pensiero di Gregorio, si coglie immediatamente la profondità che caratterizza la sua concezione del mistero del Redentore (Bouchet, "Le vocabulaire de l'union et du rapport des natures chez S. Grégoire de Nysse”, 533-582).

${ }^{44}$ Di Nissa, Contra Eunomium III, 3, 63, 7-14 (GNO II, 130, 11-18). È qui evidente la dinamicità della concezione nissena dell'unità di Cristo e della communicatio idiomatum, sottolineata da Bouchet, "Le vocabulaire de l'union et du rapport des natures chez S. Grégoire de Nysse”, 579). 
È notevole la presenza dell' $\alpha$ $\sigma 0 ́ \gamma \chi v \tau o \varsigma$ che ritornerà nel simbolo di Calcedonia e, soprattutto, l'affermazione della distinguibilità solo intellettuale delle proprietà di ciascuna natura dopo l'unione. Qui Gregorio anticipa chiaramente la formula $\theta \varepsilon \omega \rho i ́ \alpha$ uóṿn che il canone VII del II Concilio di Constantinopoli applicherà, nel 553, alle due nature di Cristo ${ }^{45}$.

In base a questo principio, bisogna considerare che la sofferenza è della carne, mentre l'atto creativo corrisponde al Verbo; infatti per natura la carne non può creare e la Divinità non può patire ${ }^{46}$. Allo stesso modo bisogna distinguere durante la vita di Cristo ciò che è segno della divinità e ciò che è segno dell'umanità: infatti non è la natura umana che risuscita Lazzaro, né quella divina che piange sul suo cadavere, né è della natura umana la moltiplicazione dei pani, né della divina la stanchezza per il cammino ${ }^{47}$. È dunque chiaro per Gregorio che:

Le ferite sono del servo nel quale è il Signore e gli onori sono, invece, del Signore, per il quale c'è il servo. Cosicché, per la congiunzione e la connaturalità diventano comuni entrambi gli effetti dell'una e dell'altra, poiché il Signore prende su di sé le lividure del servo e il servo è glorificato con l'onore del Signore. Infatti, per questa ragione, si dice che la croce è del Signore della gloria $a^{48}$ ogni lingua proclama che Gesù Cristo è il Signore, a gloria di Dio Padre (Fil 2,11).49

Laffermazione della communicatio idiomatum non può essere più chiara ed esplicita $^{50}$. Gregorio mostra, qui, che l'unione ipostatica è la ragione per la quale la croce è gloriosa: proprio poiché il Divino ha assunto il dolore e la miseria dell'uomo, l'eternità pervade l'umano, rendendo eterno il tempo dell'uomo. È questo il nucleo più intimo e profondo della teologia della storia.

L'immagine finale vuole solo esprimere icasticamente il realismo della deificazione della natura umana. Gregorio ha chiarito che la considerazione intellettuale ( $\theta \varepsilon \omega \rho i ́ \alpha)$ può distinguere dopo l'unione le diverse proprietà naturali. Cristo è sempre veramente uomo, ma uomo divinizzato, e quindi ormai privo di tutti i limiti imposti dalla limitatezza terrena: e l'aceto indica questi limiti. La distinzione tra le due

\footnotetext{
${ }^{45} \mathrm{Su}$ questo problema vedasi: Maspero, "La cristología de Gregorio de Nisa desde la perspectiva del II Concilio de Costantinopla," 359-373.

${ }^{46}$ Di Nissa, Contra Eunomium III, 3, 64, 4-65, 1 (GNO II, 130, 22-27).

${ }^{47}$ Ibíd., 3, 65, 1-8 (GNO II, 130, 27-131, 5).

${ }^{48}$ Vedasi: 1 Cor 2,8 .

${ }^{49}$ Di Nissa, Contra Eunomium III, 3, 66, 3-11 (GNO II, 131, 8-16).

${ }^{50}$ Comenta Mateo-Seco: "Al considerar la cristología nisena, en el campo de la unión hipostática, es necesario prestar atención, sobre todo, más que a la terminología usada, a la exactitud con que realiza la comunicación de idiomas" (Mateo-Seco, "Notas sobre el lenguaje cristológico de Gregorio de Nisa," 91).
} 
nature non è soppressa, invece, dalla glorificazione, ma la loro unione nella realtà dell' Uomo-Dio è così perfetta che non si possono distinguere che con la mente.

$\grave{E}$ in base a questa cristologia che Gregorio impugna l'accusa di vergognarsi della croce di Cristo, mossa da Eunomio a Basilio:

Infatti tutte e due [cioè sia noi che gli eunomiani] crediamo ugualmente

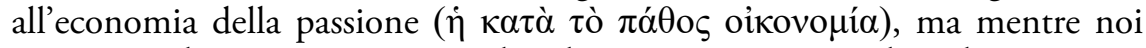
riteniamo che sia necessario rendere lo stesso onore, con il quale è onorato il Padre, al Dio che è stato manifestato mediante la croce, invece per loro la passione è un impedimento per rendere gloria all'Unigenito Dio, allo stesso modo che al Padre che lo abbia generato. ${ }^{51}$

La croce è per Gregorio la piena manifestazione della divinità del Cristo, che obbliga a rendergli onore come al Padre. La croce è, dunque, sublime rivelazione dell'amore trinitario. Ed è tale proprio perché la croce, il dolore e la sofferenza sono autentici. Infatti, quando si dice che il Cristo è luce, potenza, giustizia, vita e verità, con ragione ci si riferisce al Verbo. Però sono autentiche anche le affermazioni del dolore, del sonno, del turbamento, delle catene, del sangue, delle ferite, della sepoltura, anche se contrastano con le prime. Esse sono vere e devono riferirsi alla carne. Non si possono attribuire, invece, alla natura divina come fa Eunomio.

La discussione continua. Il ragionamento di Gregorio è stringente: Eunomio cita il passo "l'ha costituito Signore e Cristo" di At 2,36 e l'affermazione di Eb 3,1-2, dove si dice che Cristo fu costituito sacerdote, riferendo l' ̇̇ं “oín $\sigma \varepsilon v$ all' esistenza anteriore ai secoli. Ma questo non si può applicare a 2Co 5,21: "Colui che non aveva conosciuto

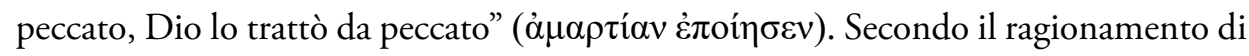

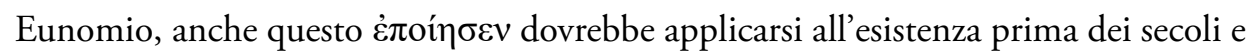
si giungerebbe all'assurdo ${ }^{52}$ :

Ma se per l'evidenza dell'assurdità Eunomio si vergognasse di questo e affermasse che l'Apostolo, dicendo lo trattò da peccato, indica l'economia negli ultimi

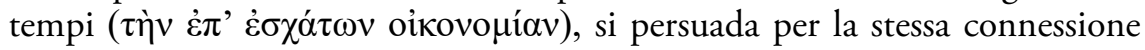

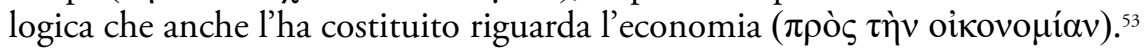

Paolo non si contraddice, perché sempre, pur proclamando l'unione dell'umano e del divino, considera in ciascun elemento ciò che gli è proprio ${ }^{54}$ :

\footnotetext{
${ }^{51}$ Di Nissa, Contra Eunomium III, 3, 30, 6-11 (GNO II, 118, 19-24).

${ }^{2}$ Ibíd., 4, 11, 1-12, 5 (GNO II, 137, 17-138, 1).

${ }^{53}$ Ibíd., 4, 12, 9-13 (GNO II, 138, 5-9).

${ }^{54}$ Ibíd., 4, 13, 9-10 (GNO II, 138, 18-10).
} 
Dunque, quando dice Dio non ha risparmiato il proprio Figlio $(\mathrm{Rm} 8,32)$, contrappone il vero Figlio agli altri figli che sono stati generati ed esaltati e divenuti ribelli, intendo dire a coloro che in forza di un comando furono fatti nascere, mentre con l'aggiunta di proprio indica la parentela secondo la natura. E perché non si attribuisca la passione della croce alla natura immacolata, con altre espressioni rettifica più chiaramente tale errore, chiamandolo mediatore fra Dio e gli uomini $(1 \mathrm{Tm} 2,5)$ e uomo e Dio, affinché, per il fatto che le due affermazioni si riferiscono all'unica realtà, si possa intendere ciò che è conveniente riguardo all'una e all'altra: l'impassibilità per la Divinità e l'economia della passione

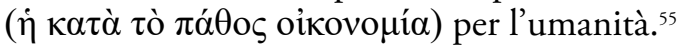

La conclusione ribadisce il principio che solo l'intelletto può distinguere in Cristo e nella sua vita terrena l'umano e il divino: "Perché è la mente (غ̇лıvoías) che divide in due quello che è divenuto una cosa sola per amore agli uomini e che viene distinto dalla ragione ( $(\lambda \hat{\gamma} \gamma \omega){ }^{\prime \prime 56}$. La mente e la ragione si affiancano ora alla $\theta \varepsilon \omega \rho i ́ \alpha$, nel ribadire la perfetta unità del Cristo.

Gregorio riafferma, dunque, che l'espressione ha costituito: "Non riguarda l'eter-

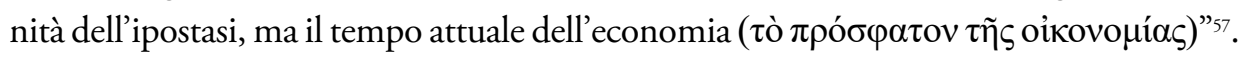
Il passo è interessante perché mostra esplicitamente che tutta la discussione tra Gregorio ed Eunomio sull'unione ipostatica verte, in realtà, sul rapporto tra eternità e tempo, sullo scandalo della presenza di Dio nella storia. Questo rapporto si articola secondo la formula del già e non ancora, come dimostra Gregorio commentando la lettera agli Ebrei:

Allo stesso modo, dunque, in questo passo si dice che "è stato fatto" sacerdote ed apostolo (Eb 3,1-2) e là [At 2,36] che "è stato fatto" Signore e Cristo: nel primo

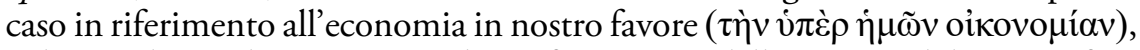
nel secondo per il mutamento e la trasformazione dell'umano nel divino. Infatti

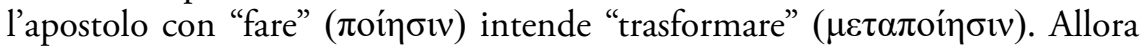
è evidente la falsità degli avversari che insolentemente usurpano i termini

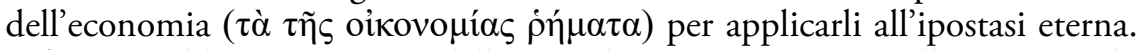
Infatti non abbiamo imparato dall'Apostolo a conoscere Cristo allo stesso modo ora e prima, perché Paolo dice così: anche se abbiamo conosciuto Cristo secondo la carne, ora non lo conosciamo più così $(2 \mathrm{Co} 5,16)$, dal momento che quella conoscenza manifestava l'economia temporanea ( mentre questa rivela l'esistenza eterna..$^{58}$

\footnotetext{
${ }^{55}$ Ibíd., 4, 14, 1-15, 1 (GNO II, 138, 23-139, 6).

${ }^{56}$ Ibíd., 4, 15, 1-3 (GNO II, 139, 6-8).

${ }^{57}$ Ibíd., 4, 17, 11-12 (GNO II, 140, 11-12).

${ }^{58}$ Ibíd., 4, 20, 1-13 (GNO II, 141, 6-18).
} 
Cristo è passibile solo nell'arco della sua vita terrena, per cui non si possono applicare gli attributi economici alla persona eterna nel tempo presente, quando siede con la sua umanità glorificata alla destra del Padre. La mutabilità tocca il Cristo solo durante la sua vita umana, cioè durante l'oỉ́ovopía, in quanto la sua natura umana è sottoposta al tempo. Ma una volta che l'incarnazione è stata portata a compimento, una volta che il Cristo è nato, ha sofferto, è morto e, infine, è risorto, la divinità pervade totalmente la sua umanità che è perfettamente elevata, conservando le sue caratteristiche proprie, ma sfuggendo al dominio del tempo:

Ma noi, avendo appreso dalla profezia il cambiamento della destra dell'Altissimo -e intendiamo che destra del Padre è la potenza di Dio che ha fatto l'universo e che è il Signore, non come una parte resa dipendente dal tutto, ma in quanto, pur essendo da lui [il Padre], è considerata nella sua propria ipostasi [il Figlio]affermiamo che la destra non è stata mutata rispetto alla natura di colui del quale è destra, né si può parlare di un suo mutamento, a parte quello che riguarda

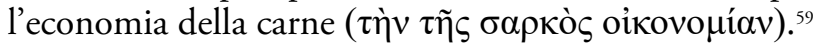

Per Eunomio la passione ha solo un significato negativo e non può essere attribuita a Dio: per lui cambiamento e peccato si identificano. Gregorio, invece, distingue nettamente la passione in quanto malattia della nostra volontà dal semplice sottostare alla legge del tempo e della mutabilità. Per questo può affermare che il Signore, che non ha conosciuto il peccato, ha preso invece parte a quelle passioni che sono così chiamate per omonimia, cioè alle condizioni naturali di ogni uomo: il sonno, la fatica, il nutrimento, il desiderio ${ }^{60}$.

E solo se si comprende l'amore di Dio per gli uomini si può accettare il mistero dell'Eterno che si assoggetta volontariamente al giogo del tempo. Infatti una malattia si cura sempre per contatto, anche se il medico nel toccare non partecipa della malattia. Così per Gregorio, in Cristo non è la malattia, cioè il peccato, che lo tocca, ma è lui che tocca la malattia e la guarisce ${ }^{6}$ :

Infatti non è la passione che tocca lui, ma è lui che tocca la malattia. Allora, se colui che mediante la sua arte procura del bene ai corpi non lo si chiama debole o malato, bensì amante degli uomini, benefattore e con gli altri nomi simili, come mai costoro, calunniando come misera e meschina l'economia

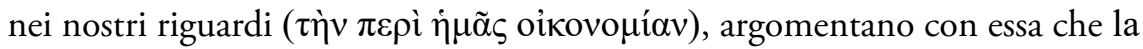
sostanza del Figlio è stata cambiata per il peggio, perché la natura del Padre sarebbe superiore alle passioni, mentre quella del Figlio non sarebbe immune

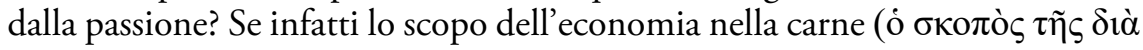

\footnotetext{
${ }^{59}$ Ibíd., 4, 24, 1-9 (GNO II, 143, 5-13).

${ }^{60}$ Ibíd., 4, 27, 1-29, 11 (GNO II, 144, 11-145, 11).

${ }^{61}$ Ibíd., 4, 31, 1-32, 1 (GNO II, 146, 2-10).
} 


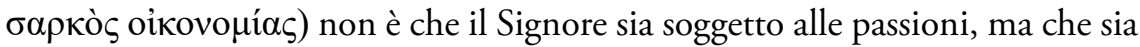
manifestato il suo amore per gli uomini, non si può dubitare che anche il Padre ama gli uomini, per cui il Padre si trova nella stessa condizione del Figlio, se si vuol considerare lo scopo ( $\sigma \kappa о \pi o ́ v){ }^{62}$

Il testo è insieme ardito e stupendo, poiché mette in evidenza, ancora una volta, che il mistero del rapporto tra il tempo e l'eterno si può comprendere solo se si crede nell'identità tra il Dio trino e l'amore. Infatti, una volta dimostrato che tutta l'oíкоvo $\mu i ́ \alpha$ non ha altro scopo e significato che la manifestazione dell'amore di Dio per l'uomo, se Eunomio accusa il Figlio di non essere Dio proprio per l'oỉкovo $\mu$ ía, e quindi per il suo amore per gli uomini, anche il Padre deve essere dichiarato inferiore.

\section{La trinità nella storia}

L'unità d'azione delle tre persone divine è uno degli assi portanti della teologia nissena. In base a questo principio Gregorio scrive:

Dunque, poiché il Figlio è potenza del Padre, tutte le opere del Figlio sono opere del Padre. Infatti è possibile presentare innumerevoli passi del Vangelo che affermano che [il Figlio] non andò incontro all'economia della passione ( $\tau \grave{v} v$

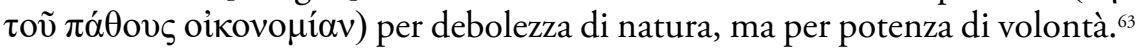

Gregorio conclude il ragionamento con forza: se ciò che è avvenuto durante la vita terrena di Cristo è un male, allora non solo il Padre deve essere escluso da ogni rapporto con questo male, ma anche il Figlio stesso. Al contrario, se si tratta di bene, bisogna ringraziare anche il Padre per la nostra salvezza. Infatti ciò che è avvenuto

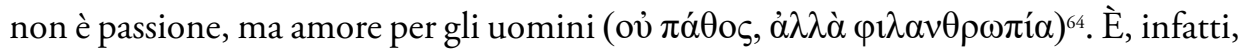
il Padre ad aver operato la nostra salvezza per mezzo del Figlio, che è la sua potenza.

Il Nisseno sa bene che tutti gli esseri presenti nella creazione vengono compresi mediante il significato dei nomi ${ }^{65}$, mentre solo la natura increata, che si crede consistere nel Padre, nel Figlio e nello Spirito Santo, è superiore ad ogni significato dei nomi ${ }^{66}$, così: "È evidente che l'appellativo Padre non indica la sostanza, ma significa la sua relazione ( $\sigma \chi \varepsilon \dot{\sigma} \sigma \mathrm{v})$ con il Figlio" ${ }^{67}$.

\footnotetext{
${ }^{62}$ Ibíd., 4, 31, 8-33, 1 (GNO II, 146, 9-22).

${ }^{63}$ Ibíd., 4, 34, 9-35, 3 (GNO II, 147, 12-16).

${ }^{64}$ Per maggiori informazioni vedere: Ibíd., 4, 35, 7-8 (GNO II, 147, 20-21).

${ }^{65}$ Ídem, Refutatio Confessionis Eunomii, 14, 5-6 (GNO II, 318, 7-8).

${ }^{66}$ Ibíd., 15, 1-3 (GNO II, 318, 15-17).

${ }^{67}$ Ibíd., 16, 4-6 (GNO II, 319, 1-3).
} 
In questo modo viene delimitato l'ambito proprio della $\theta \varepsilon o \lambda o \gamma i ́ \alpha$, che per

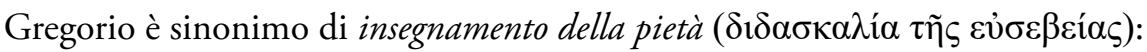

Infatti l'apprendere che Egli [Dio Padre] è l'essere che veramente è ( $\tau$ ò öv $\tau \omega \varsigma$ őv), con il quale si manifesta anche la grandezza del Figlio mediante la nozione di relazione ( $\tau \tilde{\eta} \varsigma \sigma \chi \varepsilon \tau \iota \kappa \tilde{\eta} \varsigma ~ \varepsilon ̇ v v o i ́ \alpha \varsigma)$, è il più perfetto insegnamento della vera religione, in quanto, come è detto, il Figlio allo stesso tempo indica unitamente a sé stesso lo Spirito della vita e della verità, poiché egli è vita e verità. ${ }^{68}$

La conoscenza della natura divina e delle tre persone non può essere che simultanea, poiché il Padre, il Figlio e lo Spirito non partecipano dell'unica natura, ma piuttosto si identificano con essa. Nominare una delle tre persone, vuol dire necessariamente pensare alle altre due. E non solo: vuol dire pensarle nel loro ordine di relazione intratrinitario.

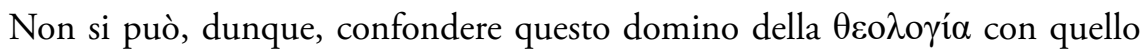
dell'oíkovouía, come riesce ad Eunomio nell'interpretare At 2,36:

Non presti attenzione per l'ordine del discorso, a cosa viene prima e cosa viene dopo in ciò che vien detto? Infatti [il testo] non dice che colui che Dio fece Signore, voi l'avete crocifisso, ma dice colui che voi avete crocifisso, questi Dio l'ha costituito Cristo e Signore, così che da ciò risulta evidente che Pietro non parla

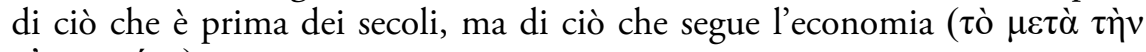
оíкоvонíav). ${ }^{69}$

Le parole della rivelazione divina sono fondamentali, il testo è sacro. Tuttavia non bisogna assolutizzare i termini, come faceva Eunomio, poiché, se il Verbo si è abbassato alla condizione umana nell'incarnazione, così anche nella Sacra Scrittura la rivelazione si abbassa al livello della comprensione umana:

Dunque, poiché queste forme della generazione sono note agli uomini,

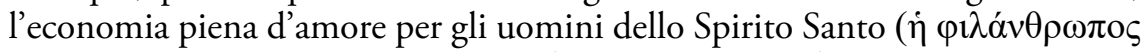

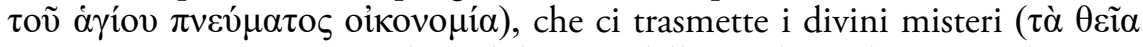
$\mu v \sigma \tau \eta ́ p 1 \alpha)$, ci insegna ciò che è al di sopra della parola mediante ciò che è per noi comprensibile. ${ }^{70}$

Per questo in molti altri passi la Scrittura utilizza espressioni antropomorfe per parlare di Dio. Così: “...per un maggior amore verso gli uomini la grazia dello Spirito

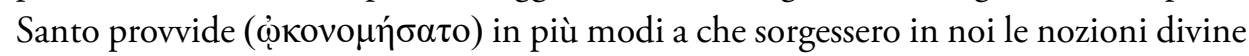
a proposito dell'Unigenito". ${ }^{71}$

\footnotetext{
${ }^{68}$ Ibíd., 17, 5-10 (GNO II, 319, 10-15).

${ }^{69}$ Idem, Contra Eunomium III, 4, 49, 4-50, 1 (GNO II, 153, 7-13).

${ }^{70}$ Ibíd., 6, 32, 1-5 (GNO II, 6-10). Il brano è parallelo a Refutatio Confessionis Eunomii, 91, 3 (GNO II, 349, 9).

${ }^{71}$ Ibíd., 6, 39, 9-11 (GNO II, 200, 3-5). Vedasi anche il brano letteralmente parallelo in Refutatio Confessionis Eunomii, 96, 1-3 (GNO II, 351, 25-352, 1).
} 
In questo passo l'unità del disegno divino sull'uomo e nella storia, culminato nell'incarnazione del Verbo, quando la Trinità stessa si è edificata nel seno della Vergine una casa nella storia, è indicata proprio con la terminologia legata a oíkovouía. Essa riunisce in un'unica espressione sia l'incarnazione che la rivelazione nella Sacra Scrittura.

Così la stessa unità del disegno divino diventa criterio esegetico imprescindibile. L'incarnazione, come centro della storia dell'uomo, rende possibile il realismo dell'interpretazione tipologica, che impedisce ogni confusione:

Ma se [Eunomio] vorrà citare anche Isaia, il quale dice che il suo nome viene chiamato angelo del gran consiglio (Is 9,5), nemmeno così potrà scalzare il nostro ragionamento. Infatti in quel passo è evidente e irrefutabile che l'economia

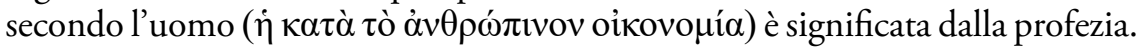
Per questo dice un bambino è nato per noi e ci è stato dato un figlio, il cui potere è sulle sue spalle ed è chiamato angelo del gran consiglio (Is 9,5). E ritengo che Davide spiegasse la costituzione del Regno mirando a questo passo, non in quanto non fosse Re, ma in quanto la condizione misera del servo, alla quale si abbassò il

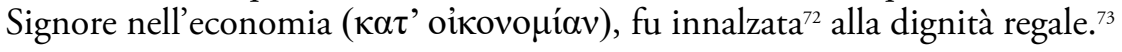

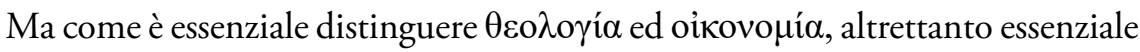
è non separarle, perché solo nella seconda si ha accesso alla prima. La confessione della fede deve necessariamente includere entrambi gli aspetti. In questo modo Gregorio traccia un meraviglioso ritratto che mostra lo scaturire della vita di Cristo dal mistero trinitario, per attrarre tutto il mondo alla comunione con il Padre nell'escatologia:

E dopo aver distinto così queste cose, anatematizzando ogni nozione eretica negli insegnamenti divini, crediamo, come ci è stato insegnato dalla parola del Signore, nel nome del Padre, del Figlio e dello Spirito Santo, confessando insieme con questa professione di fede anche l'economia messa in atto dal Signore della creazione in favore degli uomini (vं $\pi \dot{\varepsilon} \rho \tau \tilde{\omega} v \dot{\alpha} v \theta \rho \omega ́ \pi \omega v$ oíkovouíav), il quale, pur essendo di natura divina, non considerò un tesoro geloso la sua ugualianza con Dio; ma spogliò sé stesso, assumendo la condizione di servo (Fil 2,6-7) e incarnandosi nella Santa Vergine, ci riscattò dalla morte, alla quale eravamo soggetti per l'essere stati venduti dal nostro peccato, dando il suo prezioso sangue, che versò sulla croce, come prezzo del riscatto delle nostre anime. E lui, che ci ha aperto in sé stesso il cammino alla resurrezione dai morti, verrà a suo tempo nella gloria del Padre, per giudicare ogni anima secondo giustizia, quando tutti coloro che sono nei sepolcri udranno la

\footnotetext{
72 J. R. Bouchet afferma che il verbo $\alpha v \alpha \lambda \alpha \mu \beta \alpha ́ v \omega$, al passivo, assume nel linguaggio nisseno un senso d'esaltazione (Bouchet," "Le vocabulaire de l'union et du rapport des natures chez S. Grégoire de Nysse", 567).

${ }^{73}$ Di Nissa, Contra Eunomium III, 9, 39, 1-40, 1 (GNO II, 278, 22-279, 7).
} 
sua voce ed usciranno: coloro che hanno fatto il bene a una resurrezione di vita, e coloro che hanno compiuto il male ad una resurrezione di giudizio. ${ }^{74}$

È impressionante la grandiosità della visione nissena, per il quale l'oỉкovo $\mu$ í $\alpha$ sgorga dalla Trinità e dà compimento alla storia, nella resurrezione escatologica, con il ritorno al Padre. Entra qui in gioco la relazione tra la filiazione eterna di Cristo e la filiazione degli uomini al Padre in Cristo. Nell'interpretare il Padre mio e Padre vostro, detto da Cristo alla Maddalena dopo la resurrezione (Gv 20,17), Gregorio afferma:

E poiché il vero Dio e Padre è divenuto Padre e Dio della nostra primizia $(\dot{\alpha} \pi \alpha \rho \chi \tilde{\eta} \varsigma)$, non dubitiamo più che lo stesso [Dio] è divenuto anche Padre e Dio nostro, in quanto abbiamo appreso che entreremo in quello stesso cammino nel quale è entrato per primo Cristo per noi. ${ }^{75}$

Sia l'origine che il termine del movimento di exitus-reditus che contrassegna l'oíkovouía è il Padre stesso. In Cristo, nella sua umanità, ogni uomo può diventare figlio di Dio, poiché Cristo, vero Dio e vero Uomo, ha aperto il cammino. È importante sottolineare che la filiazione divina di tutti gli uomini si fonda sulla filiazione di Cristo nella sua umanità al Padre. Cristo è Figlio di Dio e figlio dell'uomo, ma la sua filiazione è unica: la sua generazione nel tempo dovette infatti essere virginale conformemente alla generazione eterna dal Padre, della quale è prolungamento ${ }^{76}$. Egli $\grave{e}$ il Figlio di Dio, egli è la filiazione; gli uomini, invece, partecipano della sua filiazione nella sua umanità. Siamo, nel modo più radicale, figli nel Figlio.

Nell'unità del disegno divino era coerente, allora, che la grazia della resurrezione fosse annunciata proprio da una donna, perché fu la donna che per prima ascoltò il serpente e peccò. È logico, dunque, che la provvidenza divina abbia previsto che fosse proprio la Maddalena la prima a conoscere e ad annunciare la resurrezione ${ }^{77}$. Quindi ci si rende facilmente conto che, fra le interpretazioni di Gv 17,20: "[quella di Eunomio] è stata forgiata per sminuire la gloria dell'Unigenito e nulla più, mentre questa [quella di Gregorio] comprende lo scopo (бко

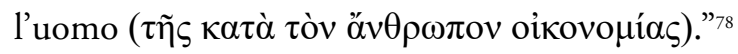

Il ragionamento del Nisseno è dinamico, quello del neoariano è statico. Per quest'ultimo il Padre deve essere ontologicamente superiore al Figlio per il semplice fatto che è rimasto immobile nella sua eternità, mentre il Figlio è entrato nel tempo

\footnotetext{
${ }^{74}$ Ídem, Refutatio Confessionis Eunomii, 18, 1-19, 7 (GNO II; 319, 16-320, 5).

75 Ídem, Contra Eunomium III, 10, 15, 7-16, 1 (GNO II, 295, 3-7).

${ }^{76}$ Maspero, "El misterio de la Virgen toda limpia en Gregorio de Nisa", 183-205.

${ }^{77}$ Per maggiori informazioni vedere a di Nissa, Contra Eunomium III, 10, 16, 1-17, 1 (GNO II, 295, 7-20).

${ }^{78}$ Ibíd., 10, 17, 5-8 (GNO II, 295, 24-27).
} 
per salvare l'uomo. Eunomio non poteva concepire un Dio onnipotente che si facesse obbediente per amore. Per Gregorio, invece, Cristo si fece obbediente realizzando il mistero della croce, umiliando sé stesso e rimediando con la sua obbedienza alla nostra disobbedienza, guarendo nella sua lividura la nostra ferita, e cancellando con la sua morte la morte di tutti gli uomini": "Allora diventa obbediente per noi, come anche

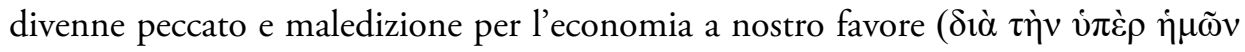
oíkovo $\mu$ íav), lui che per natura non era tale, ma lo divenne per amore degli uomini" ${ }^{80}$. Per il Nisseno, gli eretici non possono assolutamente concepire un Dio che si fa perfetto uomo:

Ma bisogna aggiungere a quanto detto ciò che essi nel discorso sull'economia ( $\dot{\varepsilon} v$

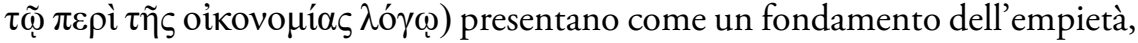
affermando che dal Signore non è stato salvato l'intero uomo, ma la metà dell'uomo, cioè il corpo. Lo scopo di tale malvagità riguardo alla dottrina è per loro mostrare che le parole più umili, che il Signore pronuncia nella sua umanità, sembrano aver avuto origine dalla Divinità stessa, per dare più forza con ciò alla bestemmia, che viene avvalorata dalla confessione stessa del Signore. Per questo dice [Eunomio]: "Colui che divenne uomo negli ultimi giorni, non assunse l'uomo in anima e corpo". Ma io, esaminata l'intera Scrittura santa e divinamente inspirata, non vi vedo scritta questa parola, cioè che Colui che creò ogni cosa assunse in sé, al momento dell'economia in favore dell'uomo

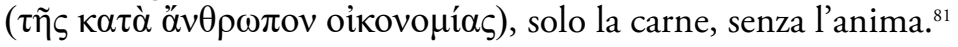

Gregorio risponde analizzando la storia e il termine della salvezza operata da Dio. Infatti non fu solo il corpo a peccare. Anzi, prima del corpo si perse l'anima, perché la trasgressione è peccato della volontà e non del corpo $^{82}$. Proprio per questa ragione, il Buon Pastore si pone sulle spalle l'intera pecora e non solo la sua pelle. ${ }^{83}$

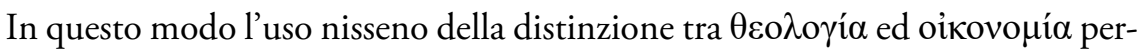
mette al grande Cappadoce di affermare, al contempo, l'immutabilità della natura divina nell'intervento nella storia e la salvezza di tutto l'uomo nella perfetta natura umana di Cristo. Proprio perché la divinizzazione ha raggiunto sia l'anima che il corpo umano, la resurrezione dai morti è divenuta possibile:

Infatti la divinità rimane sempre la stessa, sia prima della carne, sia nella carne, sia dopo la passione, essendo sempre ciò che per natura era e rimanendolo per

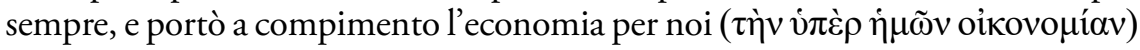

\footnotetext{
${ }^{79}$ Idem, Refutatio Confessionis Eunomii, 134, 4-13 (GNO II, 369, 28-370, 9).

${ }^{80}$ Ibíd., 134, 13-135, 1 (GNO II, 370, 9-11).

${ }^{81}$ Ibíd., 172, 1-173, 5 (GNO II, 384, 20-385, 6).

${ }^{82}$ Ibíd., 174, 1-3 (GNO II, 385, 13-15).

${ }^{83}$ Ibíd., 175, 9-176, 1 (GNO II, 386, 5-11).
} 
nella passione della natura umana, separando temporaneamente l'anima dal corpo, ma senza separarsi da nessuno dei due elementi ai quali si era unita una volta per tutte, e riunendo nuovamente ciò che era stato separato, in modo tale

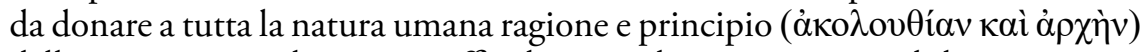
della resurrezione dai morti, affinché tutta la natura corruttibile si rivestisse di incorruttibilità e tutta la natura mortale di immortalità, essendo la nostra primizia ( $\dot{\alpha} \pi \alpha \rho \chi \tilde{\eta} \varsigma)$ trasformata in natura divina mediante l'unione con Dio

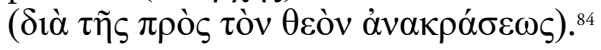

In questo modo l'affermazione di Eunomio della superiorità del Padre sul Figlio, in quanto il primo non avrebbe subito mutamento alcuno, mentre il secondo si è incarnato per la salvezza degli uomini, è radicalmente smentita proprio a partire dalla confessione del Dio uno e trino come amore e dalla considerazione del suo intervento in tutta la storia dell'uomo:

Ma non c'è, né mai ci sarà, tra i cristiani una simile idea, che sostenga che ogni bene che si trova negli esseri non ha la sua causa dal Padre. Coloro che pensano bene credono, invece, che il principale dei nostri beni è l'essere risollevati alla vita. E questo è stato realizzato mediante l'economia secondo l'uomo

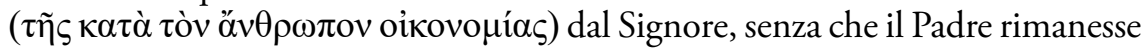
inattivo ed inoperoso, come vuole l'eresia, durante il tempo dell'economia

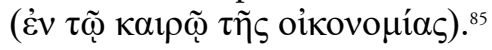

Alla difesa della perfetta umanità di Cristo, Gregorio affianca quella della sua perfetta divinità. Un grande approfondimento del mistero dell'unione ipostatica gli permette di affermare senza indugi la realtà dell'intervento del Figlio nella storia e la perfezione della redenzione da lui operata. Il ruolo centrale dato alla filiazione e l'insistenza nel considerare inseparabilmente il mistero trinitario e quello cristologico permettono al Nisseno di affermare l'intervento di tutta la Trinità nella storia stessa, che viene concepita come un unico grande e magnifico movimento di exitus-reditus, che ha come origine e come termine la casa (oíkía) del Padre.

\section{Conclusione}

Alla fine del percorso proposto emerge la specificità della riflessione nissena rispetto $\mathrm{a}$ Ireneo, Clemente, Origene ed Atanasio. Sulla scia del fratello Basilio, Gregorio supera la contrapposizione tra essere e storia che, almeno a livello espressivo, caratterizzava i tentativi che l'avevano preceduto.

\footnotetext{
${ }^{84}$ Ibíd., 179, 1-12 (GNO II, 387, 14-25).

${ }^{85}$ Ídem, Contra Eunomium III, 10, 36, 6-14 (GNO II, 303, 12-20).
} 
Gregorio di Nissa, invece, raggiunge una sintesi tra essere e storia, sintesi che è permessa, in modo forse paradossale per la prospettiva novecentesca, proprio dalla considerazione ontologica della misericordia come attributo propriamente divino che traduce ad extra la relazionalità immanente del Dio uno e trino.

Chiave di questo passaggio è la concezione dell'unione ipostatica, nella quale il tempo e l'Eterno si uniscono una volta per sempre. Così per Gregorio, la vita terrena di Cristo è una spiegazione del suo essere Figlio: Cristo, Figlio di Dio e figlio dell'uomo rende accessibile ad ogni uomo il proprio essere Dio come Figlio, rivelandolo in una storia umana. Ma tale unione tra l'Essere e la storia diventa accessibile all'uomo solo grazie alla misericordia di Dio, che nella sua immanenza più profonda è comunione di persona, in modo tale da rivelarsi nel dono reale di sé, cioè nella stessa comunione personale con il Cristo.

Da tale prospettiva, allora, l'esistenza concreta e il tempo non possono più essere d'ostacolo alla perfezione della grazia divina, perché Dio è Signore del tempo, secondo la bella formula contenuta nell' In Basilium, che può essere posta a sigillo del percorso proposto:

Così che in nulla può venir danneggiato lo scopo ( $\sigma \kappa о \pi o ́ \varsigma)$ della divina

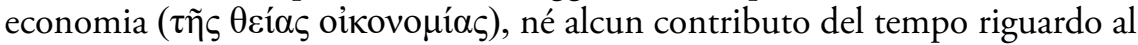
mistero ( $\pi \rho$ ò $\tau$ tò $\mu v \sigma \tau$ ́n

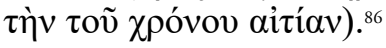

Per volere divino il tempo è il luogo della realizzazione del mistero. Per questa stessa ragione esso diventa luogo della comprensione del mistero stesso. Nel momento in cui Cristo si è fatto uomo, la storia è stata trascesa, assurgendo a dimensione essenziale di ogni autentica teologia.

\section{Riferimenti}

Bouchet, Jean-René. "Le vocabulaire de l'union et du rapport des natures chez S. Grégoire de Nysse". Revue thomiste 68 (1968): 533-582.

Brugarolas, Miguel. “La mediación de Cristo en Gregorio de Nisa." Scripta theologica 49/2 (2017): 301-326.

Cavalcanti, Elena. Studi eunomiani. Roma: Pontificium Institutum Orientalium, 1976.

Daley Brian E. "Divine Transcendence and Human Transformation: Gregory of Nyssa’s Anti-Apollinarian Christology". In Re-Thinking Gregory of Nyssa, a cura di Sarah Coakley, 67-76. Oxford: Blackwell Publishing, 2003.

${ }^{86}$ Di Nissa, Basilum fratem, GNO X/1, 114, 14-17. 
. "Heavenly Man and Eternal Christ: Apollinarius and Gregory of Nyssa on the Personnal Identity of the Savior". Journal of Early Christian Studies 10 (2002): 469-488.

Dams, Théodore. La controverse eunoméenne. Paris: Faculté de Théologie de l'Institut Catholique, 1951.

Di Nissa, Gregorio. Basilum fratem. GNO Vol. X. Tomo I. Leiden: Brill, 1990.

. Contra Eunomium. I-II. GNO Vol. I. Leiden: Brill, 1960.

. Refutatio Confessionis Eunomii. Contra Eunomium III. GNO Vol. II. Leiden: Brill, 1960.

Kopecek, Thomas. A History of Neo-arianism. Vol. I-II. Cambridge (MA): Philadelphia Patristic Foundation, 1979.

Maspero, Giulio. "Economy”. In The Brill Dictionary of Gregory of Nyssa, a cura di Giulio Maspero e Lucas Francisco Mateo-Seco, 541. Leiden-Boston: Brill, 2010. . "El misterio de la Virgen toda limpia en Gregorio de Nisa". Scripta de Maria II-1 (2004): 183-205.

- "La cristología de Gregorio de Nisa desde la perspectiva del II Concilio de Costantinopla". Scripta theologica 36 (2004): 359-373.

. "Storia e salvezza: il concetto di oikonomia fino agli esordi del III secolo". Studia Ephemeridis Augustinianum 96 (2006): 239-260.

Mateo-Seco, Lucas Francisco. "Notas sobre el lenguaje cristológico de Gregorio de Nisa”. Scripta theologica 35 (2003): 89-112.

Moreschini, Claudio. Gregorio di Nissa. Teologia trinitria. Milano: Rusconi, 1994.

Pottier, Bernard. Dieu et le Christ selon Grégoire de Nysse. Namur: Culture et Vérité, 1994.

Tixeront, Joseph. Histoire des dogmes dans l'antiquité chrétienne. II. Paris: J. Gabalda, 1909.

Vaggione, Richard Paul. Eunomius of Cyzicus and the Nicene Revolution. Oxford: Oxford University Press, 2000. . Eunomius. The Extant Works. Oxford: Clarendon Press, 1987. 